\title{
Absorption and photoluminescent spectra of dimethylaniline ethylene ketone dyes in isotropic solvents
}

\author{
M.P. Gorishnyi ${ }^{1}$, A.F. Shevchuk ${ }^{2}$, V.S. Manzhara ${ }^{1}$, A.V. Koval'chuk ${ }^{1}$, T.N. Koval'chuk ${ }^{3}$ \\ ${ }^{1}$ Institute of Physics, NAS of Ukraine, 46, prospect Nauky, 03028 Kyiv, Ukraine \\ ${ }^{2}$ Vinnitsa State Agricultural University, 3, Sonyachna str., 21008 Vinnitsa, Ukraine \\ ${ }^{3}$ V. Lashkaryov Institute of Semiconductor Physics, NAS of Ukraine, 45, prospect Nauky, 03028 Kyiv, Ukraine
}

\begin{abstract}
Absorption and photoluminescent spectra of dimethylaniline ethylene ketone dyes of two types $K_{1}$ and $K_{2}$ in a series of isotropic solvents were studied. It was shown that the photoluminescent spectra depend both on nature of solvent (formation of associates with solvent owing to hydrogen bonds between the dye and alcohol or aggregates of dye molecules) and the dye concentration (concentration decay). The frequencies of electron transitions and frequency of intramolecular fluctuation were determined, and conclusions concerning the nature of absorption bands were made.
\end{abstract}

Keywords: absorption and photoluminescent spectra, dimethylaniline ethylene ketone dye, isotropic solvent.

Manuscript received 23.11.05; accepted for publication 15.12.05.

\section{Introduction}

Interest to research liquid crystals (LCs) is substantially caused by opportunities of their practical application in electrooptical devices of different types. Essential restrictions for LC application are related with the absence of absorption bands within the visible spectral range. One way to improve the $\mathrm{LC}$ device characteristics and extend their application fields is to introduce the dyes possessing the absorption of visible radiation into the LC structure. In this connection, there arose the problem to search for dyes that effectively would fit into the LC structure. Our investigations have shown that the dimethylaniline ethylene ketone (DMAEK) dyes possess a good solubility in the LC series. In particular, in the nematic [1, 2], ferroelectric [3], and liotropic LCs, the concentration of DMAEK dyes can achieve $10 \mathrm{wt} . \%$. In this case, the solution of DMAEK dyes in nematic LCs appeared to be perspective for recording the dynamic holograms of two and four-beam geometry [4]. Revealed were essential dependences of the absorption and luminescent spectra of DMAEK dyes on both a type of used solvents and concentration, which can be connected with exhibiting the aggregation effects, formation of complexes and orientation ordering.

As in the literature there are no DMAEK molecular spectra researches, which is necessary to discuss their properties in LC matrixes, in this paper reported are the results of the experimental study and analysis of molecular spectra of DMAEK dyes in isotropic solvents.

\section{Materials and methods}

Dye absorption spectra within the wavelength range of 250 to $600 \mathrm{~nm}$ were measured using the spectrophometer "Hitachi" equipped with a personal computer. In so doing, the standard silica cuvettes of various thicknesses were used that allowed us to study the optical absorption dependence on the concentration of dyes in a solution. As solvents, we used toluene, dimethylformamide, ethanol, hexane and glycerine.

To measure luminescent spectra of solutions, we used the automated spectral setup based on a monochromator SPM-2 with photoelectric signal registration. Photoluminescence of dyes was excited by the mercury lamp ДРШ-250 radiation transmitted through the filters УФС-6 and УФС-8.

\section{Experimental results}

Structural formulae of DMAEK molecules for dyes of orange $\left(\mathrm{K}_{1}\right)$ and yellow $\left(\mathrm{K}_{2}\right)$ colors are shown in Fig. 1. The $\mathrm{K}_{1}$ molecule contains a carbonyl group $\left.{ }_{2}^{1}\right\rangle \mathrm{C}=\mathrm{O}$ in its center, to which the methylfuran radical is attached in the position 1, and dimethylaniline ethylene one - in the position 2. In the $\mathrm{K}_{2}$ molecule, the methylfuran radical is substituted for metoxybenzyl. These molecules belong to unsaturated ketones. Their fragments between furan, benzene $\left(\mathrm{K}_{1}\right)$ and benzene $\left(\mathrm{K}_{2}\right)$ rings are flat, therefore the valent electrons of $\mathrm{C}$ and $\mathrm{O}$ atoms (carbonyl group) delocalize with formation of $\pi$-systems in $\mathrm{K}_{1}$ and $\mathrm{K}_{2}$. 
<smiles>[Y]C(C)Cc1ccc(/C=C/C(=O)c2ccc(C)o2)cc1</smiles><smiles>[R1]C=Cc1ccc(N(C)C)cc1</smiles>

Fig. 1. Structure formulae of 1-methoxyfuran,2-dimethylaniline ethylene ketone $\left(\mathrm{K}_{1}\right)$ and 1-methoxybenzyl,2- dimethylaniline ethylene ketone $\left(\mathrm{K}_{2}\right)$.

Oxygen atoms of carbonyl groups form $\pi$-bonds using their $2 p_{z}$-electrons. Their unseparated pairs of $2 p_{x}$-electrons (in Fig. 1, they are marked by circles) are located in non-coupling orbitals. According to this analysis, $\pi \pi^{*}$ - and $n \pi^{*}$-states are possible in the $\mathrm{K}_{1}$ and $\mathrm{K}_{2}$ molecules.

Absorption spectra of $K_{1}$ and $K_{2}$ solutions within the range of wavelengths 280 to $500 \mathrm{~nm}$ are shown in Fig. 2. The spectrum of orange $K_{1}$ dye in benzene (curve 1) consists of two bands: an intensive longwave band with $\lambda=410 \mathrm{~nm}, \varepsilon=5 \cdot 10^{4} 1 \cdot \mathrm{mol}^{-1} \cdot \mathrm{cm}^{-1}$ and more weak shortwave one with $\lambda=315 \mathrm{~nm}, \varepsilon=1.2 \cdot 10^{4} 1 \cdot \mathrm{mol}^{-1} \cdot \mathrm{cm}^{-1}$. The spectrum of the yellow $\mathrm{K}_{2}$ dye in benzene (curve 3 ) is similar to the first one but has hypsochromical shift: the longwave band is shifted by $12 \mathrm{~nm}$, and the shortwave band - by $14 \mathrm{~nm}$.

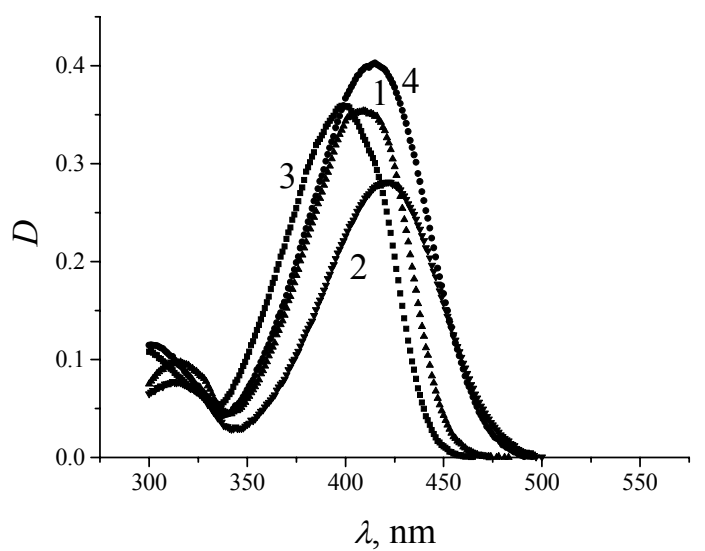

Fig. 2. Absorption spectra of $K_{1}$ and $K_{2}$ dyes in benzene $(1,3)$ and DMFA $(2,4)$. The dye concentration $C$ equals: $7.0 \cdot 10^{-6}(1) ; 5.6 \cdot 10^{-6}(2) ; 7.3 \cdot 10^{-6}(3) ; 8.1 \cdot 10^{-6}(4) \mathrm{mol} / \mathrm{l}$.
In the polar solvent, in dimethylformamide (DMFA), the longwave band of orange $\mathrm{K}_{1}$ dye is shifted bathochromically by $12 \mathrm{~nm}(0.09 \mathrm{eV})$ relatively to the band in the benzene solution spectrum, and the weak shortwave band does not change its position, but in this case its halfwidth increases and asymmetry decreases (Fig. 2, curve 2).

When passing from benzene to DMFA, the changes in the spectrum of yellow $K_{2}$ dye (curves 3 and 4 accordingly) are precisely the same as in that of orange $\mathrm{K}_{1}$ dye. The bathochromical shift of the longwave band is the main change.

For $\mathrm{K}_{2}$ dye possessing the best solubility, investigated were the absorption spectra also in other solvents as well as for various concentrations. For the concentration $C=2 \cdot 10^{-5} \mathrm{~mol} / \mathrm{l}$ in toluene, the peak of its main, longwave, absorption band appeared to have the same position $-\lambda=399 \mathrm{~nm}-$ as well as in the spectrum of benzene solutions, and in alcohol it has an essential longwave shift at $\lambda=452 \mathrm{~nm}$. With one more order increase in the concentration of $\mathrm{K}_{2}$ dye in alcohol, the longwave band prevailing in the spectrum displaces hypsochromically by $36 \mathrm{~nm}$ and decreases 5 -fold in its intensity. In glycerine, the peak of the longwave $\mathrm{K}_{2}$ absorption band occurs at $\lambda=416 \mathrm{~nm}$, i.e., coincides with the peak position in the concentrated alcohol solution spectrum.

The photoluminescent spectrum (PLS) of the $\mathrm{K}_{1}$ dye solution of the concentration $C=2 \cdot 10^{-5} \mathrm{~mol} / 1$ is a single band, the peak position of which essentially depends on a solvent: $\lambda=466 \mathrm{mn}$ in toluene and $554 \mathrm{~nm}$ in alcohol.

The PLS of $K_{1}$ dye solution in the same solvents and of the same concentration differ from those of $\mathrm{K}_{1}$ only in the peak position of the band - it lies at 460 and $538 \mathrm{~nm}$ when dissolving in toluene and alcohol, respectively (Fig. 3, curves 1 and 2). With one order increase in the concentration of $\mathrm{K}_{2}$ dye in toluene and alcohol, the position of dye luminescent bands does not change, only the concentration decay is observed (curves 3 and 5).

More essential changes are observed in the $\mathrm{K}_{2}$ PLS when it dissolves in glycerine: this spectrum has two clearly pronounced widely overlapped bands with the peak position at 489 and $570 \mathrm{~nm}$ (Fig. 3, curve 5).

When measuring the PLS, the changes of spectra occur owing to UV irradiation of solutions. These changes vary for different solvents.

For the $\mathrm{K}_{1}$ dye solution in toluene, the changes of spectra consists of reduction in the radiation intensity. The analysis of some spectra of the same solution, which have been registrated under constant conditions but after their UV irradiation for $15 \mathrm{~min}$, shows that observed is not only increase in the peak intensity of the band, but also the change of the spectrum shape: the radiation intensity at the shortwave edge of the band increases. All these spectra cross in the one - isobestic - point at $\lambda=440 \mathrm{~nm}$. In alcohol solution of $\mathrm{K}_{1}$ dye, the phototransformations do not occur. 


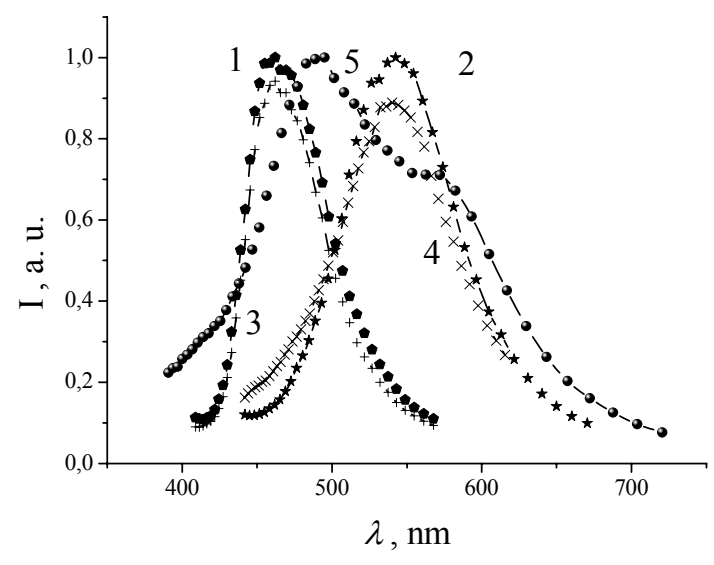

Fig. 3. $\mathrm{K}_{2}$ luminescent spectra in toluene and alcohol before $(1,2)$ and after $(3,4)$ irradiation as well as in glycerine before irradiation (5). $\mathrm{K}_{2}$ concentration in toluene and alcohol equals $2 \cdot 10^{-4} \mathrm{~mol} / \mathrm{l}$.

To obtain the additional data on the nature of phototransformations of $\mathrm{K}_{1}$ dye, the researches of its spectral changes in the course of irradiation were carried out in the binary solvent. For this aim, before measurement into the toluene solution of $\mathrm{K}_{1}$ dye $(C=$ $2 \cdot 10^{-4} \mathrm{~mol} / \mathrm{l}$ ) a small amount (less than $10^{-4} \mathrm{wt} . \%$ ) of alcohol was added. In the spectrum of unirradiated solution, observed are two overlapped bands with the peak position close to those in initial solvents: the more intensive shortwave - toluene - band with the peak at $\lambda=467 \mathrm{~nm}$ and longwave - alcohol - one with that at $\lambda=544 \mathrm{~nm}$ (Fig. 4, curve 1). On irradiation, the intensity of the first, toluene, band increases and that of the second, alcohol, decreases. The isobestic point is located at $493 \mathrm{~nm}$.

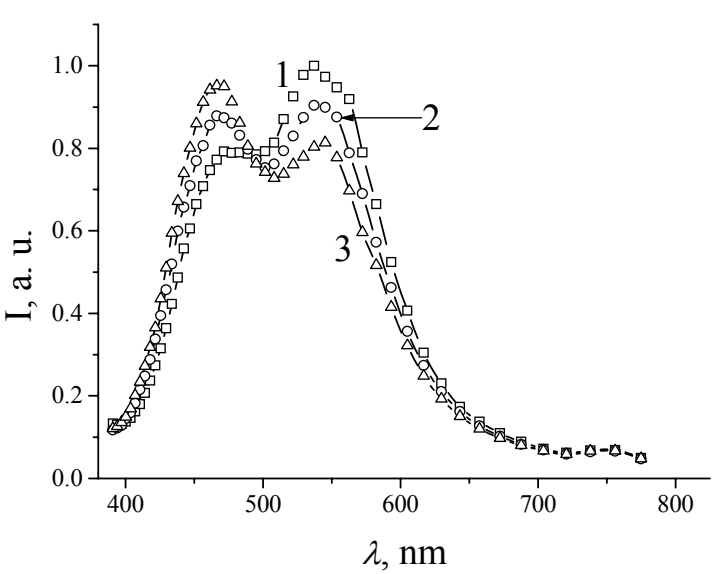

Fig. 4. Luminescent spectra of $\mathrm{K}_{2}$ solution in toluene $(C=$ $\left.=2.0 \cdot 10^{-4} \mathrm{~mol} / \mathrm{l}\right)$ with a small amount $\left(10^{-4} \mathrm{wt} . \%\right)$ of alcohol: 1 - initial condition; 2 and 3 - sequential changes of spectra in the course of UV irradiation.
The $\mathrm{K}_{2}$ dye is photostable in both alcohol and toluene solutions. For the $\mathrm{K}_{2}$ dye, the most essential changes occur under UV irradiation of its solution in glycerine (Fig. 5). In glycerine, on irradiation the longwave photoluminescent band with a peak $\lambda=570 \mathrm{~nm}$ first decreases in its intensity, and on prolonged irradiation it absolutely disappears, and instead of the band only the widely extended longwave edge of an asymmetric shortwave band remains. The shortwave band with $\lambda=$ $=489 \mathrm{~nm}$ first increases in its intensity and PLS of irradiated glycerine solutions have an isobestic point at $510 \mathrm{~nm}$. A rather photostable solution forms, the PL band of which remains almost constant in its intensity with a highly asymmetric longwave wing. But after prolonged irradiation, the band also begins to decrease in the intensity, shifts hypsochromically by $20 \mathrm{~nm}$, and a new band with $\lambda=435 \mathrm{~nm}$ arises at its shortwave edge.

\section{The analysis of experimental data}

In the work [5], to analyze spectra of aromatic molecules with a pronounced oscillation structure, the harmonic oscillation model in the Frank-Condon approximation was used in accord with the following expression:

$\frac{I_{n}}{I_{0}}=\frac{v_{n}}{v_{0}} \frac{z^{n}}{n !}$.

Here, $I_{0}$ is the intensity of a band with frequency $v_{0}, I_{n}$ is the intensity of $n$-th peak with the frequency

$v_{n}=v_{0}+n v_{k}$,

where $v_{k}$ is the frequency of fully symmetrical oscillation that dominates in the energy spectrum. The parameter $z$ is equal to the ratio of the potential energy of molecule elastic deformation on its excitation to energy of fully symmetrical oscillation

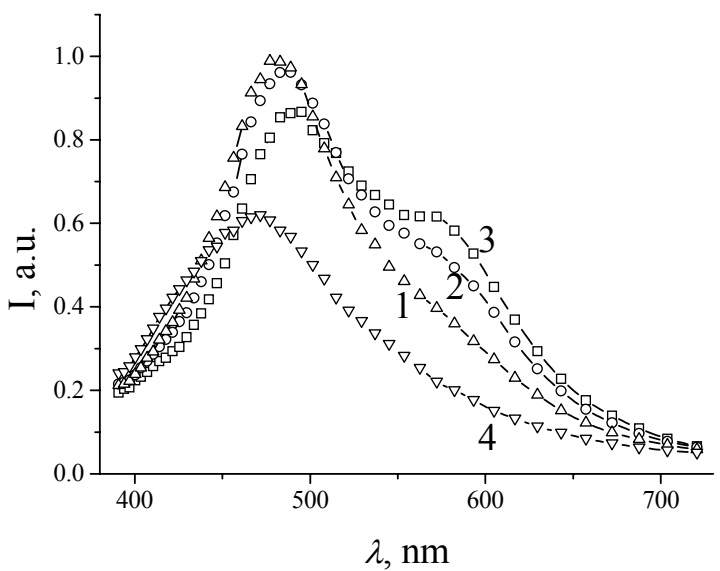

Fig. 5. Luminescent spectra of $\mathrm{K}_{2}$ solution in glycerine: 1 initial condition; 2-4 - sequential changes of spectra in the course of UV irradiation. 
$z=\frac{k(\Delta Q)^{2}}{2 h c v_{k}}$,

where $k$ is a force constant of bonds that form a molecular skeleton, $\Delta Q$ is the shift of the molecule configuration coordinate on its excitation, $h$ is Planck's constant, $c$ is the velocity of light in vacuum.

In works $[6,7]$, it was offered to use Eqs (1) and (2) also for analysis of continuous wide bands without a pronounced oscillation structure. In this method, the number of the oscillating quantum is determined as follows

$$
n=\frac{k_{1}}{k_{2}-k_{1}}
$$

where

$$
k_{1}=\frac{I_{n+1} v_{n}}{I_{n} v_{n+1}},
$$

$$
k_{2}=\frac{I_{n} v_{n-1}}{I_{n-1} v_{n}}
$$

The values $k_{1}$ and $k_{2}$ are determined experimentally. For this purpose, the values $v_{n}$ and $v_{k}$ are chosen in an arbitrary way. In the spectrum of absorption (or luminescence), measured are the intensities $I_{n-1}, I_{n}, I_{n+1}$ for three frequencies $v_{n-1}, v_{n}, v_{n+1}$ and defined are $k_{1}$ and $k_{2}$. Then, from Eq. (4) the value of $n$ is determined. By the known values of $n$ and $v_{n}$ from Eq. (2) the frequency of $0-0$ transition $\left(v_{0}\right)$ is defined.

Our estimations showed that the absorption band contour of $\mathrm{K}_{1}$ and $\mathrm{K}_{2}$ dyes solutions in toluene $(C=$ $=2 \cdot 10^{-5} \mathrm{~mol} / \mathrm{l}$ ) (Fig. 6) can be approximated with a small error by formulas [5-7] with the frequency $v_{k}=$ $=1110 \mathrm{~cm}^{-1}$ corresponding to the valent oscillation one for groups $\equiv \mathrm{C}-\mathrm{C} \equiv$ or $\rangle \mathrm{C}=\mathrm{C}=\mathrm{O}$ (ketone radical) [8]. The frequencies of peaks of these bands were determined from Eq. (2). From Eq. (4), it was found that $n=2$. Hence, it follows that the bands are caused by interaction of purely electron excited state with the second oscillator excitation. Thus, with $k=750 \mathrm{~N} / \mathrm{m}$ (the force constant for carbon bonds of anthracene molecules [6]), it yields that the derived from Eq. (3) parameter $\Delta Q=1.2 \cdot 10^{-11} \mathrm{~m}$.

The contour of luminescent bands well coordinates with that following from the theory at $v_{k}=1000 \mathrm{~cm}^{-1}$. The average value of frequency (wavenumber) of fully symmetrical skeleton oscillation of $\mathrm{K}_{1}$ and $\mathrm{K}_{2}$ molecules equals $1050 \mathrm{~cm}^{-1}$. Besides, one can assert that the system of oscillator levels for the basic and first excited electron states of the $\mathrm{K}_{1}$ and $\mathrm{K}_{2}$ molecules is the same. In case of the harmonic approximation, these levels are equidistant. The potential energy minimum of the first excited state of molecules is displaced relatively to the basic state one by $1.2 \cdot 10^{-11} \mathrm{~m}$.
Fig. 6 shows the spectra of absorption $\left(\varepsilon / \varepsilon_{\max } v=f(v)\right)$ and photoluminescence $\left(\left(I / I_{\max } v^{4}=f(v)\right)\right.$ of $\mathrm{K}_{1}$ and $\mathrm{K}_{2}$ dye solutions in toluene when $C=2 \cdot 10^{-5} \mathrm{~mol} / \mathrm{l}$, which were normalized to the maximal value. Here, $\varepsilon$ and $I$ are the values of molar extinction and PL intensity, $\varepsilon$ and $I_{\max }$ are their maximal values, $v$ is the wavenumber expressed in $\mathrm{cm}^{-1}$. Their analysis has shown that obeyed are the Stokes-Lommel law $\left(v_{\mathrm{exc}}>v_{\mathrm{Lom}}\right)$ and mirror symmetry rule for the absorption and luminescent spectra. In this case, the frequencies of purely electron transitions in $\mathrm{K}_{1}$ and $\mathrm{K}_{2}\left(v_{0}\right)$ were determined using the vertical lines that pass through the crossing points of absorption and luminescent spectra. We obtained the following values of $v_{0}: 22645 \mathrm{~cm}^{-1}(442 \mathrm{~nm})$ for $\mathrm{K}_{1}$ and $22968 \mathrm{~cm}^{-1}$ (432 nm) for $\mathrm{K}_{2}$.

The nature of molecule electron bands can be ascertained from a comparison of the spectra to the results of quantum-mechanical calculations and association of a band with the certain pair of molecular orbitals. As the calculations for the $\mathrm{K}_{1}$ and $\mathrm{K}_{2}$ molecules are unknown, their electron bands can be identified with those of functional groups to a first approximation.

In the $K_{1}$ and $K_{2}$ molecules, one can find the following conjugate chromophores: carbonyl $(>\mathrm{C}=\mathrm{O})$ and ethylene $(-\mathrm{C}=\mathrm{C}-)$ groups, dimethylaniline fragment, furan and benzene rings. Methoxygroup $(\mathrm{CHO}-)$ and methyl $\left(\mathrm{CH}_{3}-\right)$ are auxochromes. They change the intensity and characteristic band position of chromophore, into composition of which these groups do not enter.

Characteristic absorption bands of the abovementioned chromophores are summerized in the work [9]. Ethylene group, furan and benzene rings absorb in the UV region. Carbonyl group is a constituent of formaldehyde. It is characteristic for this group that there are the intensive band at $155.5 \mathrm{~nm}(\pi \rightarrow \pi *$ transition polarized along the bond $\left.\mathrm{C}=\mathrm{O}, \varepsilon=10^{4}\right)$ and the weak band caused by $n \rightarrow \pi *$-transition, at $310 \mathrm{~nm}$

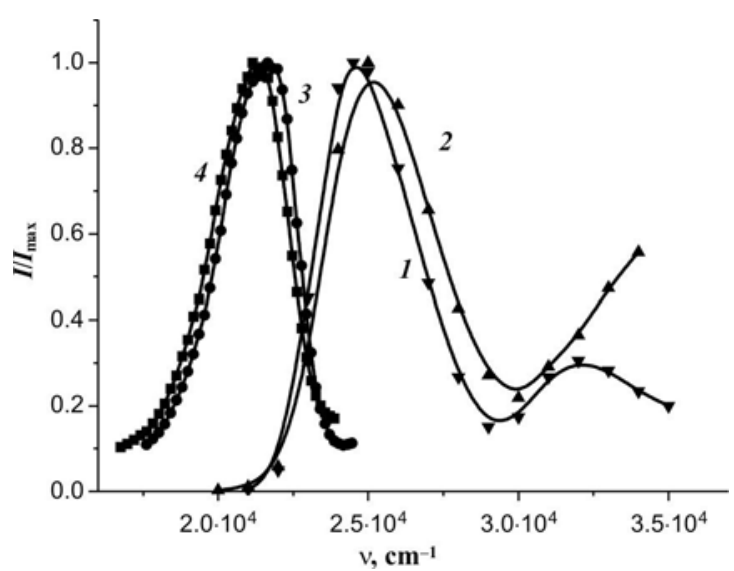

Fig. 6. Normalized to maximal value spectra of absorption $(1,2)$ and luminescence $(3,4)$ of $\mathrm{K}_{1}$ and $\mathrm{K}_{2}$ dyes. 
$\left(\varepsilon=101 \cdot \mathrm{mol}^{-1} \cdot \mathrm{cm}^{-1}\right)$ in its spectrum. Conjugation of the carbonyl and ethylene groups in $\alpha$ - and $\beta$-unsaturated ketones causes significant bathochromical $\pi \pi *$-bands in the spectral region of $220-280 \mathrm{~nm}$ without changing their intensity.

The absorption spectrum of a nitrodimethylaniline molecule consists of two bands with the peaks at $395 \mathrm{~nm} \quad\left(\varepsilon=2 \cdot 10^{4} 1 \cdot \mathrm{mol}^{-1} \cdot \mathrm{cm}^{-1}\right)$ and $229 \mathrm{~nm}(\varepsilon=$ $\left.=10^{4} 1 \cdot \mathrm{mol}^{-1} \cdot \mathrm{cm}^{-1}\right)$. By the intensity magnitudes, these two bands may be considered as stemming from the $\pi \rightarrow \pi *$-transitions. The former band is caused by excitation of the overall $\pi$-system of the molecule, and the latter is localized on the benzene ring.

The absorption spectrum of a benzylacetate phenone molecule that is the $K_{2}$ one without methoxy- and dimethylamino-groups is characterized by a wide longwave band at $370 \mathrm{~nm}\left(\varepsilon=2001 \cdot \mathrm{mol}^{-1} \cdot \mathrm{cm}^{-1}\right)$. This band passes into continuum (continuous absorption) for the wavelength $\lambda<300 \mathrm{~nm}\left(\varepsilon=10^{4} 1 \cdot \mathrm{mol}^{-1} \cdot \mathrm{cm}^{-1}\right)$ [9]. By its intensity value, the first band can be considered as a $\pi \rightarrow \pi *$-transition, and continuum - as an excitation of $\pi$ system of the overall molecule $(\lambda=300 \mathrm{~nm})$ and, in particular, benzene rings $(\lambda<300 \mathrm{~nm})$.

Intensities of absorption bands of $\mathrm{K}_{1}$ and $\mathrm{K}_{2}$ molecules (Fig. 2, curves 1-4) in the ranges of 390-420 and $280-320 \mathrm{~nm}$ equal $5 \cdot 10^{4}$ and $1.2 \cdot 10^{4} 1 \cdot \mathrm{mol}^{-1} \cdot \mathrm{cm}^{-1}$, respectively, i.e. these are $\pi \rightarrow \pi *$-transitions. Taking the above analysis into account, it is possible to assume that the former band is caused by excitation of $\pi$-system of $\mathrm{K}_{1}$ and $\mathrm{K}_{2}$ molecules. $\pi$-systems are formed owing to conjugation of the carbonyl, ethylene, and dimethylaniline groups. The second (shortwave) excitation is localized at the carbonyl and ethylene groups.

Distinction in the peak positions of longwave absorption bands of $\mathrm{K}_{1}$ and $\mathrm{K}_{2}$ solutions can be explained by different auxochrome influence of radicals of methylfuran $\left(\mathrm{K}_{1}\right)$ and methoxybenzene $\left(\mathrm{K}_{2}\right)$ on the $\pi$ systems of dye molecules.

The longwave absorption bands of the alcohol $\mathrm{K}_{1}$ and $\mathrm{K}_{2}$ solutions are shifted bathochromically by 0.15 and $0.36 \mathrm{eV}$, respectively, relatively to their positions in toluene solutions. It testifies to formation of associates between the alcohol molecules and dye ones owing to formation of hydrogen bonds between the hydroxyl and carbonyl groups. The hypsochrome shift of the peak of the longwave $\mathrm{K}_{2}$ absorption band and reduction of its intensity with one order increase in the dye concentration in alcohol solution testify to aggregation of the dye molecules. As the changes in absorption spectra occur simultaneously by I and II types [10], the question of the structure of these aggregates remains open.

PLS of $\mathrm{K}_{1}$ dyes (Fig. 4, curve 1) are caused by the dye monomer radiation $(470 \mathrm{~nm})$ and its alcohol associates (the band of $540 \mathrm{~nm}$ ). The associate concentration is small and aggregation of dye molecules is absent. Decrease in the intensity of the band at $540 \mathrm{~nm}$ on irradiation suggests that the associates destruct with formation of free $\mathrm{K}_{1}$ monomers (increase in intensity of the band at $470 \mathrm{~nm}$ ). In so doing, the total concentration of associated and quasi-free dye molecules is constant, as demonstrated by the availability of the isobestic point.

Juxtaposition of the PLS structure for $\mathrm{K}_{2}$ dye solution in glycerine to those in the other solvents, the account of absorption spectra as well as the analysis of PLS changes after UV irradiation of solutions allow to suggest that, in glycerine solution, both free $\mathrm{K}_{2}$ dye molecules and aggregated ones contribute to the PLS.

\section{Conclusions}

1. It was determined that, in the spectral region of 280 to $600 \mathrm{~nm}$, the electron bands of the studied $\mathrm{K}_{1}$ and $\mathrm{K}_{2}$ dyes in series of isotropic solvents are $\pi \pi *$-states. In so doing, the longwave absorption band is caused by excitation of the whole $\pi$-system and the shortwave one - by the carbonyl and ethylene groups of dye molecules. The frequencies of purely electron transitions are equal to $22645 \mathrm{~cm}^{-1}\left(\mathrm{~K}_{1}\right)$ and $22968 \mathrm{~cm}^{-1}\left(\mathrm{~K}_{2}\right)$. The system of oscillation levels of the basic and first excited states of dyes is identical, and, in harmonic approximation, it is determined by the frequency of intramolecular oscillation $v_{k}=$ $=1055 \mathrm{~cm}^{-1}$.

2. The solutions of $\mathrm{K}_{1}$ and $\mathrm{K}_{2}$ dyes possess photoluminescence, spectra of which depend on both nature of solvent (formation of the associates with solvent owing to hydrogen bonds between the dye and alcohol or the aggregates of dye molecules), and on the dye concentration (concentration decay).

3. It was found that photostability of dyes depends on the solvent nature and availability of impurities, with which the dye molecules can form associates.

Work was executed at financial support of the budgetary theme 1.4.1 B/109 of Institute of Physics, NAS of Ukraine.

\section{References}

1. A.V. Kukhta, Electroluminescence of thin films of organic compounds (Review) // J. Appl. Spectr. 70, p. 165-194 (2003).

2. O.V. Kovalchuk, DC current in dye-doped liquid crystals // Mol. Cryst. and Liquid Cryst. 361, p.157163 (2001).

3. A.V. Koval'chuk, Relaxation processes and charge transport across liquid crystal-electrode interface // J. Phys.: Condens. Matter. 13, p. 10333-10345 (2001).

4. A.F. Shevchuk, D.A. Naiko, M.N. Pivnenko, A.V. Koval'chuk, The Influence of strongly dissociative impurity on "anomalous" high-frequency conductivity of smectic phases // Ukr. Fiz. Zhurn. 47, p. 464-468 (2002) (in Ukrainian).

5. A. Miniewicz, K. Komorovska, O.V. Koval'chuk, J. Vanchanen, J. Sworakowski, M.V. Kurik, Studies of photorefractive properties of a novel dye-doped 
nematic liquid crystal system // Adv. Mater. Opt. Electron. 10, p. 55-67 (2000).

6. E.F. McCoy, I.G. Ross, Electronic states of aromatic hydrocarbons: the Frank-Condon principle and geometrics in excited states // Austral. J. Chem. 15, p. 573-587 (1962).

7. V.P. Klochkov, Contour of absorption and emission bands of composite molecules // Optika i spektroskopiya 19, p. 337-344 (1965) (in Russian).

8. V.P. Klochkov, S.M. Korotkov, Determination of electron transition frequency in continuous spectra of absorption and emission // Ibid. 20, p. 582-588 (1966) (in Russian).

9. C.N. Banwell, Fundamentals of molecular spectroscopy. Mir, Moscow (1985) (in Russian).

10. O.V. Sverdlova, Electron spectra in organic chemistry. Khimiya, Leningrad (1985) (in Russian).

11. R.A. Friedel, M. Orchin, Ultraviolet spectra of aromatic compounds. John Wiley and Sons Inc., New York (1951).

12. L.V. Levshin, A.M. Saletskiy, Luminescence and its application. Moscow State University, Moscow (1989) (in Russian). 